\title{
Review of: "Criss-cross hemostatic suture in nephron sparing open surgery"
}

\section{Carlos Ameri}

Potential competing interests: The author(s) declared that no potential competing interests exist.

I want to congratulate the authors for this interesting surgical technique.

Despite reporting only one case, they listed their series which makes the results more valuable.

From my point of view, the "criss-cross" hemostatic suture is valid only for polar tumors as it was described in the series of Ameri et al (New technique for nephron-sparing surgery in polar tumours. A modification of the Kim technique. doi:10.1016/j.acuro.2017.01.010)

It would be interesting to compare results between both series.

Futhermore, the authors should add the RENAL score in order to compare with others series (results and complications).

Finally, a cohort study would be more interesting and report their series and not only one case. 